\title{
Scattering of first and second sound waves by quantum vorticity in superfluid Helium
}

\author{
Christophe Coste \\ Laboratoire de Physique, École Normale Supérieure de Lyon \\ 46, Allée d’Italie,69364 Lyon Cedex 07, France \\ FERNANDO LUND \\ Departamento de Física, Facultad de Ciencias Físicas y Matemáticas \\ Universidad de Chile, Casilla 487-3, Santiago, Chile
}

\begin{abstract}
We study the scattering of first and second sound waves by quantum vorticity in superfluid Helium using two-fluid hydrodynamics. The vorticity of the superfluid component and the sound interact because of the nonlinear character of these equations. Explicit expressions for the scattered pressure and temperature are worked out in a first Born approximation, and care is exercised in delimiting the range of validity of the assumptions needed for this approximation to hold. An incident second sound wave will partly convert into first sound, and an incident first sound wave will partly convert into second sound. General considerations show that most incident first sound converts into second sound, but not the other way around. These considerations are validated using a vortex dipole as an explicitely worked out example.
\end{abstract}




\section{Introduction}

The interaction of collective excitations with superfluid vortices has been a subject of intense research ever since the concept of mutual friction was introduced [1, 2, 3]. For reviews see [4, 5]. Recent problems have included the question of the inertial mass of a vortex [6, 7], the nature of the force on a moving vortex[8], possible generalizations to other systems that support vortices and collective excitations [9], as well as approaches based on the nonlinear Schrödinger equation [10, 11].

As an alternative to an approach based on a microscopic description of a superfluid, it is possible to consider the well established equations of two fluid hydrodynamics 12]. These equations describe, among others, two modes of flow: One of them, obtained by linearizing the equations around a static, uniform state, is sound, both first and second. Another can be associated with superfluid vortical flow, in which the only dynamical quantity is the superfluid velocity. The corresponding vorticity is concentrated within thin filaments, whose circulation is quantized in units of $h / m$. This last fact comes into the two-fluid equations as an ad-hoc assumption. Because of the nonlinearity of the hydrodynamic equations, these two modes interact, and as a result a sound wave that impinges on a superfluid vortex will be scattered. This paper is devoted to a study of this effect. Previous work [13 studied the scattering of second sound waves by quantum vorticity very near the lambda point, at temperatures such that the superfluid density is very small compared to the normal fluid density. Earlier, Pitaevskii[14] calculated the scattering of first sound by a quantum vortex in two dimensions in a Born approximation, and Fetter 15 in an exact form. Other nonlinear acoustic effects, related to the interaction of the sound waves with themselves, have also been studied [16, 17].

In this paper we study the scattering of sound (both first and second) by quantum vorticity within the framework of two fluid hydrodynamics, in a first Born 
approximation in three space dimensions. The reasoning follows earlier ideas that were developped in classical hydrodynamics 18, that have been succesfully tested in experiment [19]. We hope that succesfully implementing the ideas of the present paper may lead to a new experimental probe to study the properties of quantum vorticity.

\section{Equations of the problem}

In this section we derive the equations needed to study the interaction of sound waves with quantum vorticity under the following assumptions: sound frequency is high compared with the inverse of all relevant time scales associated with the vortex motion,as well as with time scales associated with viscous and thermal relaxation effects; velocity amplitudes associated with sound are small compared to velocities associated with the vortices, and the latter are small compared to the speed of both first and second sound (even near the vortex cores); finally, thermal expansion effects are assumed to be negligible. Our analysis will be based in the phenomenological two-fluid model, in which Helium II, of density $\rho$, is regarded as a mixture of normal fluid, of density $\rho_{n}$, and a superfluid, of density $\rho_{s}$, with $\rho=\rho_{s}+\rho_{n}$. The flow of this system is described by normal and superfluid velocity fields $\mathbf{V}_{n}$ and $\mathbf{V}_{s}$. A characteristic feature of this model is that it predicts that small disturbances away from equilibrium values obey a wave equation, and that when the coefficient of thermal expansion is small enough, there are two modes of propagation: one ("first sound") corresponds to density fluctuations as in normal fluids; the other ("second sound") corresponds to temperature fluctuations. The speed of propagation of second sound waves depends strongly on temperature and it vanishes at the Lambda point [4. The dynamics of the two-fluid model is described by the following set of nonlinear equa- 
tions that quantify the conservation of mass, the conservation of linear momentum, the fact that the vorticity associated with the superfluid velocity is concentrated within vortex cores, and the fact that entropy is transported by the normal fluid only 12$]$ :

$$
\begin{aligned}
\frac{\partial \rho}{\partial t}+\nabla \cdot \mathbf{J} & =0 \\
\frac{\partial J_{i}}{\partial t}+\nabla_{j} \Pi_{i j} & =0 \\
\frac{\partial \mathbf{V}_{s}}{\partial t}+\left(\mathbf{V}_{s} \cdot \nabla\right) \mathbf{V}_{s} & =-\nabla \mu+\mathbf{f}, \\
\frac{\partial(\rho S)}{\partial t}+\nabla \cdot\left(\rho S \mathbf{V}_{n}\right) & =0
\end{aligned}
$$

where

$$
\begin{aligned}
\mathbf{J} & =\rho_{s} \mathbf{V}_{s}+\rho_{n} \mathbf{V}_{n}, \\
\Pi_{i j} & =p \delta_{i j}+\rho_{n} V_{n i} V_{n j}+\rho_{s} V_{s i} V_{s j}, \\
d \mu & =\frac{1}{\rho} d p-S d T-\frac{\rho_{n}}{2 \rho} d W^{2}, \\
\mathbf{f} & =-\frac{B \rho_{n}}{2 \rho} \boldsymbol{\omega}_{\boldsymbol{s}} \times\left(\hat{\omega}_{s} \times \mathbf{W}\right)-\frac{B^{\prime} \rho_{n}}{2 \rho} \boldsymbol{\omega}_{\boldsymbol{s}} \times \mathbf{W}
\end{aligned}
$$

where $p, S$ and $T$ are the pressure, entropy per unit mass, and temperature, respectively, $\mathbf{W} \equiv \mathbf{V}_{n}-\mathbf{V}_{s}, \boldsymbol{\omega}_{\boldsymbol{s}} \equiv \nabla \times \mathbf{V}_{s}$ is the superfluid vorticity, and $\hat{\omega}_{s} \equiv \boldsymbol{\omega}_{\boldsymbol{s}} /\left|\boldsymbol{\omega}_{\boldsymbol{s}}\right|$. $B$ and $B^{\prime}$ are the usual mutual friction coefficients. Thermal and viscous diffusivity effects have been neglected assuming the sound frequency to be large. Eqn. (3) contains terms that involve only the interaction of a superfluid vortex with itself ("self-induction term"); they have been discarded because they do not couple to the sound waves. The entropy equation (位) has terms cubic in the velocity; they have been omitted because they will be shown to be negligible below. 
We describe the flow as the sum of fast irrotational "sound" (v) and slow incompressible "vortical" $\left(\mathbf{U}_{s}\right)$ parts:

$$
\mathbf{V}=\mathbf{v}+\mathbf{U}_{s}, \quad \nabla \times \mathbf{v}=0, \quad \nabla \cdot \mathbf{U}_{s}=0, \quad v \ll U_{s}
$$

Note that the sound component $\mathbf{v}$ contains both normal and superfluid components. The vortical component $\mathbf{U}_{s}$ is a permanent superfluid flow, the equilibrium velocity field; it is a solution of the equations obtained by considering constant densities $\rho_{0 n}$ and $\rho_{0 s}$, temperature $T_{0}$ and entropy $S_{0}$, as no normal fluid flow is involved $\left(\mathbf{V}_{n}=0\right)$, and from which $\nabla \cdot \mathbf{U}_{s}=0$ follows as a consequence. This flow arises because vortex filaments move under their mutual and self-induction, possible external flows and appropriate boundary conditions. The decomposition given in Eq. (9) is unique when boundary conditions for $\mathbf{U}_{s}$ are specified. For instance, that it vanish sufficiently fast at infinity so that a scattering formulation makes sense. It is simple to check that under these conditions the dynamics is completely governed by the equation

$$
\frac{\partial \mathbf{V}_{s}}{\partial t}+\left(\mathbf{V}_{s} \cdot \nabla\right) \mathbf{V}_{s}=-\frac{1}{\rho_{0}} \nabla p+\frac{\rho_{0 n}}{2 \rho_{0}} \nabla \mathbf{V}_{s}^{2}+\mathbf{f}_{0}
$$

where $\mathbf{f}_{0}$ is the mutual friction force $\mathbf{f}$ evaluated at constant densities. Taking the curl of Eq. (10) yields an evolution equation for the superfluid vorticity, from which the (incompressible) superfluid velocity may be deduced. Taking the divergence of Eq. (10) yields an elliptic equation for the pressure in terms of the superfluid velocity. Thus, just as in classical incompressible hydrodynamics, the pressure is not a dynamical variable, but it is determined at each instant of time by the current value of the superfluid velocity.

The flow $\mathbf{v}$ includes both first and second sound, and consists of small deviations of density $\rho^{\prime}$, temperature $T^{\prime}$, entropy $S^{\prime}$ and pressure $p^{\prime}$ away from their equilibrium values (note that, due to the flow $\mathbf{U}_{s}$, the equilibrium value $p_{0}$ of the pressure is not 
a constant). The interaction between the two modes, sound and vortical, is obtained by linearizing the equations around the vortical solution $\mathbf{U}_{s}$. The result on equation (11) is

$$
\frac{\partial \rho^{\prime}}{\partial t}+\rho_{s}^{0} \nabla \cdot \mathbf{v}_{s}+\rho_{n}^{0} \nabla \cdot \mathbf{v}_{n}=-\left(\mathbf{U}_{s} \cdot \nabla\right) \rho_{s}^{\prime}
$$

and we note that the right hand member is linear in $\mathbf{U}_{s}$. Applying the same procedure on (2) leads to

$$
\rho_{s}^{0} \frac{\partial v_{s i}}{\partial t}+\rho_{n}^{0} \frac{\partial v_{n i}}{\partial t}+\nabla_{i} p^{\prime}=-\frac{\partial\left(\rho_{s}^{\prime} U_{s i}\right)}{\partial t}-\nabla_{j}\left(\rho_{s}^{\prime} U_{s i} U_{s j}+\rho_{s}^{0} U_{s j} v_{s i}+\rho_{s}^{0} U_{s i} v_{s j}\right)
$$

in wich a quadratic term, $\nabla_{j}\left(\rho_{s}^{\prime} U_{s i} U_{s j}\right)$, appears. Let us compare this term with $\left(\partial\left(\rho_{s}^{\prime} U_{s i}\right) / \partial t\right)$ :

$$
\begin{gathered}
\frac{\partial\left(\rho_{s}^{\prime} U_{s i}\right)}{\partial t}=\underbrace{U_{s i} \frac{\partial \rho_{s}^{\prime}}{\partial t}}_{\simeq \nu_{0} \rho_{s}^{\prime} U_{s}}+\underbrace{\rho_{s}^{\prime} \frac{\partial U_{s i}}{\partial t}}_{\simeq(1 / \Delta t) \rho_{s}^{\prime} U_{s}} \\
\nabla_{j}\left(\rho_{s}^{\prime} U_{s i} U_{s j}\right)=\underbrace{U_{s i} U_{s j} \nabla_{j} \rho_{s}^{\prime}}_{\simeq k_{0} \rho_{s}^{\prime}\left(U_{s}\right)^{2}}+\underbrace{\rho_{s}^{\prime} U_{s j} \nabla_{j} U_{s i}}_{\simeq(1 / L) \rho_{s}^{\prime}\left(U_{s}\right)^{2}}
\end{gathered}
$$

where $\nu_{0}$ is the wave frequency, $k_{0}$ its wavenumber, $\Delta t$ a typical time scale for the vortical flow, and $L$ a typical length scale. We assume $\nu_{0} \gg 1 / \Delta t$, so that the second term in the r.h.s. of (13) is negligible; the wave probes the vortical flow on typical scales equal to its wavelength, so that $k_{0} \simeq 1 / L$, and

$$
\frac{\left|\nabla_{j}\left(\rho_{s}^{\prime} U_{s i} U_{s j}\right)\right|}{\left|\left(\partial\left(\rho_{s}^{\prime} U_{s i}\right) / \partial t\right)\right|} \simeq \frac{U_{s}}{c_{i}} \ll 1
$$

where $c_{i}$ is either the first $(i=1)$ or the second $(i=2)$ sound velocity. However, the last inequality is not obviously verified, because the velocity $U_{s}$ may take large values near the vortex core. Sound scattering effects, as most wave mechanics effects, 
are most dramatic when interference effects are dominant. This occurs when the wavelength is comparable to the length scale of the structure responsible for the scattering. Consequently, sound scattering will "see" structures with a typical size equal to the wavelength of the sound wave, say $\lambda_{i}$. At this distance from the core of a vortex line, $U_{s} \simeq \hbar /\left(m_{H e} \lambda_{i}\right)$, where $m_{H e}$ is the mass of an helium atom. Thus if $\nu_{0}$ is the frequency of the wave, we have

$$
\frac{U_{s}}{c_{i}} \ll 1 \Longleftrightarrow \nu_{0} \ll \frac{m_{H e} c_{i}^{2}}{\hbar} \approx\left\{\begin{array}{l}
510^{12} \mathrm{~Hz} \text { (first sound) } \\
810^{9} \mathrm{~Hz} \text { (second sound) }
\end{array}\right.
$$

The condition is easy to realize for first sound waves, and clearly compatible with our previous requirement that the time scale of the sound wave should be much smaller than that of thermal and viscous diffusion effects. For the second sound wave, the numerical value is calculated for $c_{2} \approx 10 \mathrm{~m} / \mathrm{s}$, which is the minimum value of the second sound velocity between $1.75 \mathrm{~K}$ and $2.15 \mathrm{~K}\left(c_{2}=0\right.$ at $T_{\lambda}$, but it is only very near the $\lambda$-point that it becomes less than $10 \mathrm{~m} / \mathrm{s}$ ). Once again, the required inequality is easy to fulfill, so that it is consistent to neglect the term which is quadratic in $U_{s}$ in (12) whatever the nature of the incident wave. This term however, will be responsible for the spontaneous generation of sound, a topic that is outside the scope of the present work (see [21] for the situation in classical hydrodynamics).

Let us now turn to the linearization of (3). Two new terms appear. The first one reads $\left(\rho^{\prime} / \rho_{0}\right) \nabla p_{0}$; but from the Bernouilli formula we know that $\nabla p_{0} \simeq \rho_{0} U_{s}^{2}$. This term vanishes in the case of a second sound wave, because in this case [20] $\rho^{\prime}=0$, and for a first sound wave it is of order $U_{s} / c_{1}$ in comparison of linear terms in $U_{s}$, and may thus be neglected.

The other term comes from the linearization of the mutual friction force $\mathbf{f}$, and 
we have to calculate $\left(\rho_{n}^{\prime} U_{s}^{2}\right) /\left(\rho_{n}^{0} U_{s} v_{s}\right)$. In the case of a first sound wave, this ratio is obviously of order $U_{s} / c_{1}$, thus negligible under the same approximations as before. For a second sound wave, one needs to be more careful. In that case [20], $\rho^{\prime} \approx 0$, but $\rho_{s}^{\prime}=\left(\partial \rho_{s} / \partial T\right)_{0} T^{\prime} \neq 0$, and $T^{\prime} \simeq-\left(c_{2} / S_{0}\right) v_{s}$ where $c_{2}$ is the velocity of second sound waves. It is possible to show that the quadratic terms are negligible at sufficiently high temperatures [however, not too close to the $\lambda$-point; see the discussion following (16)] ; in this limit, the thermodynamic quantities are accurately given by the roton part of the excitation spectrum [12]. We get

$$
\frac{\rho_{n}^{\prime} U_{s}^{2}}{\rho_{n}^{0} U_{s} v_{s}}=\left(\frac{\rho_{0}}{\rho_{n}^{0}}\right)\left(\frac{\Delta_{r} / T_{0}-1 / 2}{\Delta_{r} / T_{0}+3 / 2}\right)\left(\frac{c_{2} U_{s}}{3\left(k_{B} T_{0} / \pi_{0}\right)^{2}}\right),
$$

where $k_{B}$ is the Boltzmann's constant, $\Delta_{r}$ is the energy of the rotons and $\pi_{0}$ their impulsion. As soon as $T_{0} \geq 1.75 \mathrm{~K}$, the ratio in the r.h.s. of (17) is less than $1 \%$. To obtain this estimate, we assumed $U_{s} \simeq 10^{-2} c_{2}$ as before, so that the quadratic terms are all negligible, with the same validity range as before. The equation derived from (3) thus reads

$$
\begin{gathered}
\frac{\partial \mathbf{V}_{s}}{\partial t}+\frac{1}{\rho_{0}} \nabla p^{\prime}-S_{0} \nabla T^{\prime}=-\frac{\rho_{s}^{0}}{\rho_{0}}\left[\left(\mathbf{v}_{s} \cdot \nabla\right) \mathbf{U}_{s}+\left(\mathbf{U}_{s} \cdot \nabla\right) \mathbf{v}_{s}\right] \\
-\frac{\rho_{n}^{0}}{\rho_{0}}\left[\left(\mathbf{v}_{n} \cdot \nabla\right) \mathbf{U}_{s}+\left(\mathbf{U}_{s} \cdot \nabla\right) \mathbf{v}_{n}\right]-\left[1-\frac{B^{\prime}}{2}\right] \frac{\rho_{n}^{0}}{\rho_{0}}\left(\mathbf{v}_{n}-\mathbf{v}_{s}\right) \times \boldsymbol{\omega}_{s} \\
-\frac{B \rho_{n}^{0}}{2 \rho_{0}} \boldsymbol{\omega}_{s} \times\left[\hat{\omega}_{s} \times\left(\mathbf{v}_{n}-\mathbf{v}_{s}\right)\right] .
\end{gathered}
$$

As for the last equation, the linearization of (4), it doesn't contain a source term, and reads

$$
S_{0} \frac{\partial \rho^{\prime}}{\partial t}+\rho_{0} \frac{\partial S^{\prime}}{\partial t}+\rho_{0} S_{0} \nabla \cdot \mathbf{v}_{n}=0
$$


The absence of source terms in this equation is due to the neglect of diffusive effects, and moreover comes from the fact that the mutual friction terms are all cubic in the velocities, hence quadratic in $\mathbf{U}_{s}$, so that we can neglect them under the same assumptions that led to equations (12) and (18).

In liquid helium, the coefficient of thermal expansion $\beta=-(1 / \rho) \partial \rho / \partial T$ is extremely small, and may be neglected. It simplifies greatly the equation of sound propagation, because in this limit the free propagation of first and second sound are completely decoupled. Indeed, after some algebra, using the fact that the sound wave flow is irrotational and that the vortical flow is divergence-free, one finds for the equations of propagation

$$
\left(\frac{1}{c_{1}^{2}} \frac{\partial^{2}}{\partial t^{2}}-\Delta\right) p^{\prime}=F_{1}
$$

where

$$
F_{1} \equiv \rho_{s}^{0}\left\{\Delta\left(\mathbf{U}_{s} \cdot \mathbf{v}_{s}\right)+\nabla \cdot\left[\boldsymbol{\omega}_{s} \times \mathbf{v}_{s}+\mathbf{U}_{s}\left(\nabla \cdot \mathbf{v}_{s}\right)\right]\right\}
$$

and

$$
\left(\frac{1}{c_{2}^{2}} \frac{\partial^{2}}{\partial t^{2}}-\Delta\right) T^{\prime}=F_{2}
$$

where

$$
\begin{aligned}
F_{2} \equiv & \frac{1}{\rho_{0} S_{0}}\left\{\left(\mathbf{U}_{s} \cdot \nabla\right)\left(\frac{\rho_{0}}{\rho_{s}^{0}} \frac{\partial \rho_{s}^{\prime}}{\partial t}+\rho_{s}^{0} \nabla \cdot \mathbf{v}_{s}\right)-\rho_{n}^{0}\left[\Delta\left(\mathbf{U}_{s} \cdot \mathbf{v}_{n}\right)+\nabla \cdot\left(\boldsymbol{\omega}_{s} \times \mathbf{v}_{n}\right)\right]\right. \\
& \left.-\left[1-\frac{B^{\prime}}{2}\right] \rho_{n}^{0} \nabla \cdot\left[\left(\mathbf{v}_{n}-\mathbf{v}_{s}\right) \times \boldsymbol{\omega}_{\boldsymbol{s}}\right]-\frac{B \rho_{n}^{0}}{2} \nabla \cdot\left[\boldsymbol{\omega}_{\boldsymbol{s}} \times\left(\hat{\omega}_{s} \times\left(\mathbf{v}_{n}-\mathbf{v}_{s}\right)\right)\right]\right\}
\end{aligned}
$$

Equations (20) and (21) are accurate to first order in $v_{s} / U_{s}, U_{s} / c_{\mathrm{i}}(\mathrm{i}=1,2)$, and $\nu_{0} \delta t$, in the temperature range $[1.75,2.15] K$. To zeroth order $\left(F_{1}=F_{2}=0\right)$ they respectively describe the usual equations for free first and second sound propagation. 


\section{Scattering of sound waves by vorticity}

Equations (20) and (21) can both be written as integral scattering equations,

$$
S O U N D_{\text {scatt. }}=S O U N D_{\text {inc. }}+G_{i} * F_{i}
$$

where $S O U N D$ is either a pressure wave, in the case of first sound, or a temperature wave, in the case of second sound; $i=1,2$ corresponding to first or second sound: $G_{i}$ is the retarded Green function for the wave equation, with vanishing boundary conditions at infinity,

$$
G_{i}=G_{i}\left(\mathbf{x}-\mathbf{x}^{\prime}, t-t^{\prime}\right)=\left(4 \pi\left|\mathbf{x}-\mathbf{x}^{\prime}\right|\right)^{-1} \delta\left(t-t^{\prime}-c_{i}^{-1}\left|\mathbf{x}-\mathbf{x}^{\prime}\right|\right)
$$

and the star $*$ denotes a space-time convolution. The scattering problem is ready to be solved using the Born approximation, which consists of injecting in $F_{1}$ and $F_{2}$ the values of $\rho_{n}^{\prime}, \mathbf{v}_{s}$ and $\mathbf{v}_{n}$ given by the incident (first or second) sound wave. In either case, both $F_{1}$ and $F_{2}$ are different from 0 , so that a second sound wave illuminating a vortex leads to a scattered first sound wave, and conversely.

\subsection{Scattered second sound from incident second sound}

We consider the scattering of an incident plane temperature wave.

$$
T^{\prime}=T_{i}^{\prime} \cos \left(k_{02} x-\nu_{0} t\right)=\frac{T_{i}^{\prime}}{2} e^{i\left(k_{02} x-\nu_{0} t\right)}+C C,
$$

where $C C$ stands for "complex conjugate", and $k_{02} \equiv \nu_{0} / c_{2}$ is the relevant wave vector for a second sound wave of frequency $\nu_{0}$. It is easy to show [20] that the velocity field is given by

$$
\mathbf{v}_{s}=\mathbf{v}_{\mathrm{i}} e^{i\left(k_{02} x-\nu_{0} t\right)}+C C, \quad \mathbf{v}_{n}=-\frac{\rho_{s}^{0}}{\rho_{n}^{0}} \mathbf{v}_{s}, \quad \mathbf{v}_{\mathrm{i}}=-\frac{S_{0} T_{i}^{\prime}}{2 c_{2}} \hat{\imath}
$$


where $\hat{\imath}$ is a unit vector in the incident direction. Moreover, we have

$$
\rho_{s}^{\prime}=\left(\frac{\partial \rho_{s}}{\partial T}\right)_{\rho}^{0} T^{\prime}+\left(\frac{\partial \rho_{s}}{\partial \rho}\right)_{T}^{0} \rho^{\prime} \approx\left(\frac{\partial \rho_{s}}{\partial T}\right)_{\rho}^{0} T^{\prime}
$$

where the superscript " 0 " indicates that all partial derivatives are taken at equilibrium, and the last approximation is justified by the smallness of the coefficient of thermal expansion $\beta([2])$. It is thus possible to show that $F_{2}=-\nabla \cdot \mathbf{D}_{2}$, where the vector $\mathbf{D}_{2}$ is given by

$$
\begin{gathered}
\mathbf{D}_{2}=\underbrace{-\frac{\left(\partial \rho_{s} / \partial T\right)_{\rho}^{0}}{\rho_{s}^{0} S_{0}} \frac{\partial \rho_{s}^{\prime}}{\partial t} \mathbf{U}_{s}}_{\equiv(a)}+\underbrace{\frac{\rho_{n}^{0}}{\rho_{0} S_{0}}\left[\nabla\left(\mathbf{U}_{s} \cdot \mathbf{v}_{n}\right)+\boldsymbol{\omega}_{s} \times \mathbf{v}_{n}+\mathbf{U}_{s}\left(\nabla \cdot \mathbf{v}_{n}\right)\right]}_{\equiv(b)} \\
+\underbrace{\left[1-\frac{B^{\prime}}{2}\right] \frac{1}{S_{0}} \mathbf{v}_{s} \times \boldsymbol{\omega}_{s}}_{\equiv(c)}+\underbrace{\frac{B}{2 S_{0}} \boldsymbol{\omega}_{s} \times\left(\hat{\omega}_{s} \times \mathbf{v}_{s}\right)}_{\equiv(d)}
\end{gathered}
$$

Let $T_{(a)}^{\prime}$ be the contribution of the term $(a)$; the detailed calculation of this term uses the following steps:

$$
\begin{aligned}
\frac{T_{(a)}^{\prime}}{T_{i}^{\prime}} & =\frac{\left(\frac{\partial \rho_{s}}{\partial T}\right)_{\rho}^{0}}{2 \rho_{s}^{0} S_{0}} \int \mathrm{d} t^{\prime} \int \mathrm{d}^{3} x^{\prime} G_{2}\left(\mathbf{x}-\mathbf{x}^{\prime}, t-t^{\prime}\right) \nabla \cdot\left[\mathbf{U}_{s}\left(\mathbf{x}^{\prime}, t^{\prime}\right) e^{-i \nu_{0} t^{\prime}}\left(-i \nu_{0}\right) e^{i \mathbf{k}_{02} \cdot \mathbf{X}^{\prime}}\right] \\
& =\frac{c_{2}\left(\frac{\partial \rho_{s}}{\partial T}\right)_{\rho}^{0}}{2 \rho_{s}^{0} S_{0}}\left(-i k_{02}\right) \int \mathrm{d}^{3} x^{\prime} \widetilde{G}_{2}\left(\mathbf{x}-\mathbf{x}^{\prime}, \nu\right) \nabla \cdot\left(\widetilde{\mathbf{U}}_{s}\left(\mathbf{x}^{\prime}, \nu-\nu_{0}\right) e^{i \mathbf{k}_{02} \cdot \mathbf{X}^{\prime}}\right) \\
& =\frac{c_{2}\left(\frac{\partial \rho_{s}}{\partial T}\right)_{\rho}^{0}}{2 \rho_{s}^{0} S_{0}} \frac{\left(-i k_{02}\right)}{2}\left[\int \mathrm{d}^{3} x^{\prime} \widetilde{G}_{2} \nabla \cdot\left(\widetilde{\mathbf{U}}_{s} e^{i \mathbf{k}_{02} \cdot \mathbf{X}^{\prime}}\right)-\int \mathrm{d}^{3} x^{\prime} \nabla \widetilde{G}_{2} \cdot \widetilde{\mathbf{U}}_{s} e^{i \mathbf{k}_{02} \cdot \mathbf{X}^{\prime}}\right] \\
& =\frac{T_{0} S_{0}\left(\frac{\partial \rho_{s}}{\partial T}\right)_{\rho}^{0}}{C \rho_{n}^{0}} \frac{i \nu \pi^{2} e^{i \nu r / c_{2}}}{r c_{2}^{2}} \frac{1}{2(1-\cos \theta)}(\hat{\imath} \times \hat{r}) \cdot \widetilde{\omega}_{s}\left(\mathbf{q}_{2 \rightarrow 2}, \nu-\nu_{0}\right)
\end{aligned}
$$

where 


$$
\widetilde{\omega}_{s}(\mathbf{q}, \nu)=\frac{1}{(2 \pi)^{4}} \int \mathrm{d} t \mathrm{~d}^{3} x e^{i(\nu t-\mathbf{q} \cdot \mathbf{x})} \boldsymbol{\omega}_{\boldsymbol{s}}(\mathbf{x}, t)
$$

is the space-time Fourier transform of the vorticity, and

$$
\mathbf{q}_{2 \rightarrow 2} \equiv \frac{\nu}{c_{2}} \hat{r}-\frac{\nu_{0}}{c_{2}} \hat{\imath}
$$

is the momentum transfer, with $\hat{r}$ a unit vector in the direction of observation, and $\hat{\imath}$ a unit vector in the direction of propagation of the incident wave. In the last line of (28), we introduced the scattering angle $\theta$, the heat capacity $C$, and we also used the asymptotic behavior at large distance of $\widetilde{G}_{i}$, which we quote here for reference

$$
\left\{\begin{array}{l}
\left.\widetilde{G}_{i} \stackrel{x \gg x^{\prime}}{{ }^{i \nu r} / c_{i}} / 4 \pi r\right) e^{i \nu \hat{r} \cdot \mathbf{X}^{\prime} / c_{i}}, \\
\nabla \widetilde{G}_{i} \stackrel{x \gg x^{\prime}}{{ }^{\prime}}\left(i \nu / c_{i}\right)\left(e^{i \nu r / c_{i}} / 4 \pi r\right) e^{i \nu \hat{r} \cdot \mathbf{X}^{\prime} / c_{i}}(-\hat{r}) \\
\Delta \widetilde{G}_{i} \stackrel{x \gg x^{\prime}}{\simeq}\left(i \nu / c_{i}\right)^{2}\left(e^{i \nu r / c_{i}} / 4 \pi r\right) e^{i \nu \hat{r} \cdot \mathbf{X}^{\prime} / c_{i}}
\end{array}\right.
$$

Moreover, we used the fact that the difference between the frequencies of the incident and scattered wave is of the order of the typical frequency of $\mathbf{U}_{s}(\mathbf{x}, t)$, thus much smaller than $\nu_{0}$, so that $\nu / c_{2} \approx \nu_{0} / c_{2}$. Note that this assumption is very easy to fulfill, if one consideres (16).

The source terms are linear in $\mathbf{U}_{s}$, which is completely characterized by its vorticity, being a divergenceless field; the scattered field should thus be expressed as a function of the vorticity, and more precisely of its Fourier transform. The dependence of $T_{(a)}^{\prime}$ on $(\hat{\imath} \times \hat{r}) \cdot \widetilde{\omega}_{s}$ was to be expected, because it is the only possible scalar expression depending both on the direction of propagation of the incident wave $\hat{\imath}$, on the direction of observation $\hat{r}$ and on the vorticity. The only term in (27), leading to a different behavior, is $(\mathrm{d})$ which is second order in vorticity. The calculation of 
the contributions of the remaining terms in (27) requires only integrations by parts, and leads to the following result :

$$
\frac{T_{2 \rightarrow 2}^{\prime}(\mathbf{x}, \nu)}{T_{i}^{\prime}}=\frac{i \nu \pi^{2} e^{i \nu r / c_{2}}}{r c_{2}^{2}}\left[H_{c}(\theta)(\hat{\imath} \times \hat{r}) \cdot \widetilde{\omega}_{s}-\frac{B}{2} \hat{r}_{i} \hat{\imath}_{j} \widetilde{\Omega}_{i j}\right]
$$

where the Fourier transforms are both taken at the point $\left(\mathbf{q}_{2 \rightarrow 2}, \nu-\nu_{0}\right)($ see (30)), and where

$$
H_{c}(\theta) \equiv\left(1-\frac{B^{\prime}}{2}\right)-\frac{1}{2(\cos \theta-1)} \frac{T_{0} S_{0}}{C \rho_{n}^{0}}\left(\frac{\partial \rho_{s}}{\partial T}\right)_{\rho}^{0}-\frac{\rho_{s}^{0}}{\rho_{0}} \frac{\cos \theta}{\cos \theta-1}
$$

and

$$
\Omega_{i j} \equiv \frac{\omega_{i} \omega_{j}}{\omega}-\omega \delta_{i j}
$$

The terms that are multiplied by $B$ and $\left(1-B^{\prime} / 2\right)$ have already been calculated by Lund \& Steinberg [13], but in their approximation scheme there should be a factor of $\rho_{n}^{0} / \rho_{0}$, which they dropped because in their approximations it is very close to 1 . Our calculation shows that term to be an artifact of the approximations used, and it dissapears in our more accurate scheme. The second term in the r.h.s. of (33) was also obtained in [13]. The last one is a correction of their result, when $\rho_{s}$ is not negligible; it is exactly the same term (up to the factor $\rho_{s}^{0} / \rho_{0}$ ) that is found for the scattering of ordinary sound by a vortex, in a classical fluid [18]. This was to be expected since the corresponding source term comes from the terms quadratic in velocity of the conservation of momentum equation.

\subsection{Scattered first sound from incident second sound}

A second sound temperature wave, as we have seen, couples to the superfluid vortical velocity field; it causes vibrations of the vortices, and those vibrations generate a scattered second sound wave, but also a first sound wave : The vortical velocity 
field couples the two differents acoustic modes in Helium II, even in our approximation, $\beta=0$, in which their respective equations of free propagation are completely decoupled.

The source $F_{1}$ in $(20)$ is a sum of three different contributions, and they finally have to be expressed as a function of $(\hat{\imath} \times \hat{r}) \cdot \widetilde{\omega}_{s}$ only, as we explained before. This is obvious for the term $\nabla \cdot\left(\boldsymbol{\omega}_{s} \times \mathbf{v}_{s}\right)$, but not for the two other terms. We may proceed in the following way to show that it is indeed the case. After a Fourier transformation in time, we have to calculate

$$
\begin{aligned}
& \int \mathrm{d}^{3} x^{\prime} \widetilde{G}_{2}\left[\Delta\left(\widetilde{\mathbf{U}}_{s} \cdot \mathbf{v}_{s}\right)+\nabla \cdot\left(\widetilde{\mathbf{U}}_{s}\left(\nabla \cdot \mathbf{v}_{s}\right)\right)\right]= \\
& \int \mathrm{d}^{3} x^{\prime}\left[\Delta \widetilde{G}_{2}\left(\widetilde{\mathbf{U}}_{s} \cdot \mathbf{v}_{s}\right)-\alpha \nabla \widetilde{G}_{2} \cdot\left(\widetilde{\mathbf{U}}_{s}\left(\nabla \cdot \mathbf{v}_{s}\right)\right)+(1-\alpha) \widetilde{G}_{2}\left(\widetilde{\mathbf{U}}_{s} \cdot \nabla\right)\left(\nabla \cdot \mathbf{v}_{s}\right)\right]
\end{aligned}
$$

where $\alpha$ is, for the time being, an unknown parameter. We have integrated by parts the integral containing the Laplacian operator, and we have split the second one, using integration by parts for the term in factor of $\alpha$, and $\nabla \cdot \mathbf{U}_{s}=0$ in order to simplify the last term. The r.h.s. of (35) takes the form

$$
\frac{e^{i \nu r / c_{1}}}{4 \pi r} \frac{i \nu}{c_{1}} \int \mathrm{d}^{3} x^{\prime} X\left(\mathbf{x}^{\prime}, \nu-\nu_{0}\right) e^{-i \mathbf{q}_{2 \rightarrow 1} \cdot \mathbf{X}^{\prime}}
$$

where

$$
\mathbf{q}_{2 \rightarrow 1} \equiv \frac{\nu}{c_{1}} \hat{r}-\frac{\nu_{0}}{c_{2}} \hat{\imath}
$$

and $X$, which is a function of $\left(\mathbf{x}^{\prime}, \nu-\nu_{0}\right)$ like $\widetilde{\mathbf{U}}_{s}$, is given by

$$
X \equiv i\left[k_{1}\left(\widetilde{\mathbf{U}}_{s} \cdot \mathbf{v}_{\mathrm{i}}\right)+\alpha\left(\hat{r} \cdot \widetilde{\mathbf{U}}_{s}\right)\left(\mathbf{v}_{\mathbf{i}} \cdot \mathbf{k}_{02}\right)+\frac{1-\alpha}{k_{1}}\left(\mathbf{k}_{02} \cdot \widetilde{\mathbf{U}}_{s}\right)\left(\mathbf{v}_{\mathbf{i}} \cdot \mathbf{k}_{02}\right)\right]
$$

where $k_{1} \equiv \nu / c_{1}$. The difference between the frequencies of the incident and scattered wave is of the order of the typical frequency of $\mathbf{U}_{s}(\mathbf{x}, t)$, thus much smaller than $\nu_{0}$, so that $k_{1} \approx \nu_{0} / c_{1}$. However, $k_{02}=\nu_{0} / c_{2}$ and the sound velocities of the 
two acoustic modes are very different, so that we do not have the simplifying assumption $k_{02} \approx k_{1}$, as in the scattering of sound by vorticity in simple fluids 18, or as in the previous case of second sound scattering from incident second sound. But, introducing an unknown coefficient $\alpha$ allows us to choose it in order that $X$ be proportional to $(\hat{\imath} \times \hat{r}) \cdot\left(\mathbf{q}_{2 \rightarrow 1} \times \widetilde{\omega}_{s}\right)$; indeed, expressing the equation

$$
X\left(\mathbf{x}^{\prime}, \nu-\nu_{0}\right)=\mathcal{C}(\hat{\imath} \times \hat{r}) \cdot\left[\mathbf{q}_{2 \rightarrow 1} \times \widetilde{\omega}_{s}\left(\mathbf{x}^{\prime}, \nu-\nu_{0}\right)\right]
$$

with $\mathcal{C}$ an (as yet) unknown coefficient of proportionality, in terms of the components of the relevant vectors in the scattering plane,

$\mathbf{k}_{02}=\left(\begin{array}{c}k_{02} \\ 0 \\ 0\end{array}\right), \mathbf{k}_{1}=\left(\begin{array}{c}k_{1} \cos \theta \\ k_{1} \sin \theta \\ 0\end{array}\right), \hat{r}=\left(\begin{array}{c}\cos \theta \\ \sin \theta \\ 0\end{array}\right), \mathbf{v}_{\mathbf{i}}=\left(\begin{array}{c}v^{i} \\ 0 \\ 0\end{array}\right), \widetilde{\mathbf{U}}_{s}=\left(\begin{array}{c}\tilde{U}_{s}^{x} \\ \tilde{U}_{s}^{y} \\ \tilde{U}_{s}^{z}\end{array}\right)$

the elimination of $\tilde{U}_{s}^{x}$ and $\tilde{U}_{s}^{y}$ leads to a linear system of two equations, for the two unknowns $\alpha$ and $\mathcal{C}$, whose solution is

$$
\mathcal{C}=\frac{1+\left(c_{2} / c_{1}\right)}{2\left(c_{2} / c_{1}\right) \cos \theta-1-\left(c_{2} / c_{1}\right)^{2}}
$$

Note that if we let $c_{1}=c_{2}$, we recover the expression valid in simple fluids with only one kind of acoustic wave [18]. Using (41), we get the pressure field scattered by a vortical region illuminated by second sound,

$$
\frac{\tilde{p}_{2 \rightarrow 1}^{\prime}(\mathbf{x}, \nu)}{\rho_{s}^{0} S_{0} T_{i}^{\prime}}=-\frac{i \nu \pi^{2} e^{i \nu r / c_{1}}}{r c_{2} c_{1}} \Pi(\theta)(\hat{\imath} \times \hat{r}) \cdot \widetilde{\omega}_{s}\left(\mathbf{q}_{2 \rightarrow 1}, \nu-\nu_{0}\right),
$$

where $\mathbf{q}_{2 \rightarrow 1}$ is defined by (37) and $\Pi(\theta)$ is given by

$$
\Pi(\theta) \equiv \frac{2\left(c_{2} / c_{1}\right) \cos \theta}{2\left(c_{2} / c_{1}\right) \cos \theta-1-\left(c_{2} / c_{1}\right)^{2}} .
$$


The reader is reminded that this equation, together with (32), is valid for temperatures in the range $[1.75,2.15] K$, under the general assumptions of section 2.

\subsection{Scattered first sound from incident first sound}

We consider now the scattering of an incident plane pressure wave.

$$
p^{\prime}=p_{i}^{\prime} \cos \left(k_{01} x-\nu_{0} t\right)=\frac{p_{i}^{\prime}}{2} e^{i\left(k_{01} x-\nu_{0} t\right)}+C C,
$$

where $C C$ stands for "complex conjugate", and now $k_{01}$ is the relevant wave vector for first sound waves, $k_{01} \equiv \nu_{0} / c_{1}$, in contrast with sections 3.1 and 3.2. It is easy to show [20] that the velocity field is given by

$$
\mathbf{v}_{s} \approx \mathbf{v}_{\mathrm{i}} e^{i\left(k_{01} x-\nu_{0} t\right)}+C C, \quad \mathbf{v}_{n}=\mathbf{v}_{s}, \quad \mathbf{v}_{\mathrm{i}}=\frac{p_{i}^{\prime}}{2 \rho_{0} c_{1}} \hat{\imath}
$$

Moreover, we have

$$
\rho^{\prime}=\frac{p_{i}^{\prime}}{2 c_{1}^{2}} e^{i\left(k_{01} x-\nu_{0} t\right)}+C C
$$

where the contribution of terms proportional to the coefficient of thermal expansion $\beta$ has been neglected.

First sound scattering from the vortical flow is given by the source term in the equation of propagation for the pressure, (20). This term, apart from a factor

$\rho_{s}^{0}$ instead of $\rho_{0}$, is identical to the corresponding one in normal fluids [18], hence the scattered pressure field is exactly the same as for ordinary sound scattering by vorticity in normal fluids, and reads

$$
\frac{\tilde{p}_{1 \rightarrow 1}^{\prime}(\mathbf{x}, \nu)}{p_{i}^{\prime}}=\frac{\rho_{s}^{0}}{\rho_{0}} \frac{i \nu \pi^{2} e^{i \nu r / c_{1}}}{r c_{1}^{2}} \frac{\cos \theta}{\cos \theta-1}(\hat{\imath} \times \hat{r}) \cdot \widetilde{\omega}_{s}\left(\mathbf{q}_{1 \rightarrow 1}, \nu-\nu_{0}\right) .
$$


where

$$
\mathbf{q}_{1 \rightarrow 1} \equiv \frac{\nu}{c_{1}} \hat{r}-\frac{\nu_{0}}{c_{1}} \hat{\imath}
$$

Note that the approximation $\nu / c_{1} \approx k_{01}$ is valid here, as in ordinary fluids. This ensures the same scattering of (ordinary) sound waves in both cases.

\subsection{Scattered second sound from incident first sound}

Let us consider now the second sound scattered by a vortical flow illuminated by a first sound wave. In a first sound wave, neglecting the coefficient of thermal expan$\operatorname{sion} \beta$ [20], we have $\mathbf{v}_{s}=\mathbf{v}_{n}$, so that the mutual friction terms do not contribute to the scattering. Moreover, equation (26) has to be replaced by

$$
\rho_{s}^{\prime}=\left(\frac{\partial \rho_{s}}{\partial T}\right)_{\rho}^{0} T^{\prime}+\left(\frac{\partial \rho_{s}}{\partial \rho}\right)_{T}^{0} \rho^{\prime} \approx\left(\frac{\partial \rho_{s}}{\partial \rho}\right)_{T}^{0} \rho^{\prime}
$$

because $T^{\prime} \approx 0$ for a first sound wave. The relevant expression of $F_{2}$ in (21) now reads $F_{2}=-\nabla \cdot \mathbf{D}_{2}^{\prime}$, with $\mathbf{D}_{2}^{\prime}$ given by

$$
\mathbf{D}_{2}^{\prime}=\frac{1}{\rho_{0} S_{0}}\left[1-\frac{\rho_{0}}{\rho_{s}^{0}}\left(\frac{\partial \rho_{s}}{\partial \rho}\right)_{T}^{0}\right] \frac{\partial \rho}{\partial t} \mathbf{U}_{s}+\frac{\rho_{n}^{0}}{\rho_{0} S_{0}}\left[\nabla\left(\mathbf{U}_{s} \cdot \mathbf{v}_{n}\right)+\boldsymbol{\omega}_{s} \times \mathbf{v}_{n}+\mathbf{U}_{s}\left(\nabla \cdot \mathbf{v}_{n}\right)\right]
$$

The calculation now proceeds just as in section 3.2; the wave vector coming from the Green function $G_{2}, \mathbf{k}_{2} \equiv\left(\nu / c_{2}\right) \hat{r}$, is very different in amplitude from the incident wave vector, $\mathbf{k}_{01}=\left(\nu_{0} / c_{1}\right) \hat{\imath}$, even if $\nu \approx \nu_{0}$, because the sound velocities $c_{1}$ and $c_{2}$ are very different. We thus have to use the same trick as in equation (35), and the result reads

$$
\widetilde{T}_{1 \rightarrow 2}^{\prime}(\mathbf{x}, \nu)=p_{i}^{\prime} \Theta(\theta) \frac{i \nu \pi^{2} e^{i \nu r / c_{2}}}{r c_{2} c_{1}}(\hat{\imath} \times \hat{r}) \cdot \widetilde{\omega}_{s}\left(\mathbf{q}_{1 \rightarrow 2}, \nu-\nu_{0}\right) .
$$

where $\theta$ is the scattering angle, the scattering wavevector $\mathbf{q}_{1 \rightarrow 2}$ is 


$$
\mathbf{q}_{1 \rightarrow 2} \equiv \frac{\nu}{c_{2}} \hat{r}-\frac{\nu_{0}}{c_{1}} \hat{\imath}
$$

and $\Theta(\theta)$ is given by

$$
\begin{aligned}
\Theta(\theta) \equiv \frac{\left(c_{2} / c_{1}\right)}{1+\left(c_{2} / c_{1}\right)^{2}-2\left(c_{2} / c_{1}\right) \cos \theta} \times \\
\quad \times\left\{\frac{1}{\rho_{0} c_{1} c_{2}} \frac{S_{0} \rho_{s}^{0}}{C \rho_{n}^{0}}\left[\frac{\rho_{0}}{\rho_{s}^{0}}\left(\frac{\partial \rho_{s}}{\partial \rho}\right)_{T}^{0}-1\right] T_{0}+\frac{2 \rho_{n}^{0}}{\rho_{0}^{2} S_{0}} \cos \theta\right\} .
\end{aligned}
$$

The reader is reminded that this equation, together with (47), is valid for all temperatures, under the general assumptions of section 2 .

\section{Estimates and example}

In this section we estimate the relative energy scattered in each acoustic mode in the case of an incident first and second sound wave. Then we choose as an example target a rectilinear vortex dipole. The simplest possible flow is that of a rectilinear vortex; however in this case, the scattered waves (32) and (47) diverge in the forward scattering direction $\theta \rightarrow 0$, and this divergence disappears with a vortex dipole 222.

The time-averaged energy density of a sound wave is

$$
\bar{E}=\rho_{s}^{0} \overline{v_{s}^{2}}+\rho_{n}^{0} \overline{v_{n}^{2}}
$$

where the overline denotes a time average over one period of oscillation [12]. Far away from the target, the scattered wave may be approximated by a plane wave, so that the energy flow is given by $I=\bar{E} c$, where $c$ is the velocity of either a first or second sound wave. To calculate the superfluid and normal velocity fields, we use the following set of equations

$$
\rho_{s}^{0} \frac{\partial \mathbf{V}_{s}}{\partial t}+\rho_{n}^{0} \frac{\partial \mathbf{V}_{n}}{\partial t}+\nabla p^{\prime}=0
$$




$$
\rho_{n}^{0} \frac{\partial}{\partial t}\left(\mathbf{V}_{n}-\mathbf{V}_{s}\right)+\rho_{0} S_{0} \nabla T^{\prime}=0
$$

They are obtained by linearizing and combining (2) and (5) in the first case, and (7), (3) and (2) in the second case. The energy flow for each kind of wave thus reads

$$
\begin{aligned}
& I_{1}=\frac{1}{\rho_{0}} \overline{\left|\nabla p^{\prime}\right|^{2}} \frac{c_{1}}{\nu^{2}} \quad \text { (first sound) } \\
& I_{2}=\frac{\rho_{s}^{0} \rho_{0} S_{0}^{2}}{\rho_{n}^{0}} \overline{\left|\nabla T^{\prime}\right|^{2}} \frac{c_{2}}{\nu^{2}} \quad \text { (second sound) }
\end{aligned}
$$

The gradients in (57) and (58) must be evaluated at leading order in $1 / r$ for consistency.

Define $\widetilde{\omega}_{s \perp}$, the component of superfluid vorticity perpendicular to the scattering plane, through the relation

$$
\sin \theta \quad \widetilde{\omega}_{s \perp}\left(\mathbf{q}_{\mathrm{i} \rightarrow \mathrm{j}}\right) \equiv(\hat{\imath} \times \hat{r}) \cdot \widetilde{\omega}_{s}\left(\mathbf{q}_{\mathrm{i} \rightarrow \mathrm{j}}\right)
$$

and, for the sake of argument, consider the following estimates:

$$
\begin{aligned}
H_{c}(\theta) & \sim 1 \\
\Pi(\theta) & \sim c_{2} / c_{1} \\
\Theta(\theta) & \sim \frac{c_{c} \rho_{n}^{0}}{c_{1} \rho_{0}^{2} S_{0}}
\end{aligned}
$$

and ignore the possible contribution of the second term on the right hand side of (32). In this case, use of (32) and (42) leads to the following estimate for the ratio of energy flux scattered as first sound to energy flux scattered as second sound for an incident second sound wave:

$$
\frac{I_{2 \rightarrow 2}}{I_{2 \rightarrow 1}} \sim \frac{\rho_{0}^{2}}{\rho_{s}^{0} \rho_{n}^{0}}\left(\frac{c_{1}}{c_{2}}\right)^{5} \frac{\left|\widetilde{\omega}_{s \perp}\left(\mathbf{q}_{2 \rightarrow 2}\right)\right|^{2}}{\left|\widetilde{\omega}_{s \perp}\left(\mathbf{q}_{2 \rightarrow 1}\right)\right|^{2}} .
$$

Similarly, use of (47) and (51) leads to the following estimate for the ratio between the energy flux scaterred as first sound and the energy flux scattered as second sound 
for an incident first sound wave:

$$
\frac{I_{1 \rightarrow 1}}{I_{1 \rightarrow 2}} \sim \frac{\rho_{s}^{0}}{\rho_{n}^{0}} \frac{c_{2}}{c_{1}} \frac{\left|\widetilde{\omega}_{s \perp}\left(\mathbf{q}_{1 \rightarrow 1}\right)\right|^{2}}{\left|\widetilde{\omega}_{s \perp}\left(\mathbf{q}_{1 \rightarrow 2}\right)\right|^{2}} .
$$

It is apparent that, for both incident sound modes, most of the energy will be scattered as second sound by superfluid vorticity. A plausible explanation of this effect is as follows: the scattering is due to the vortex being jiggled by the incoming sound, and the corresponding reemission of sound. Now, the vortex involves superfluid motion only, so that most of the sound reemited will involve the superfluid velocity being out of phase with the normal component velocity, as appropriate for second sound waves.

We now work out a specific example to validate this result.

\subsection{Scattering by a vortex dipole}

Consider a vortex dipole oriented along the $x_{2}$-axis, build of two counter-rotating vortices of circulation $\pm \Gamma$, at distances $\pm a$ from the $x_{1}$-axis; the induced flow is not stationary, and the dipole moves along the $x_{1}$-axis at the constant speed $V_{d}=$ $\Gamma /(4 \pi a)$ [23. For wavelengths $\lambda$ large compared to the vortex separation $a$ as well as vortex core radius, the vorticity field can be approximated as

$$
\boldsymbol{\omega}_{\boldsymbol{s}}=\Gamma 2 a \delta^{\prime}\left(x_{2}\right) \delta\left(x_{1}-V_{d} t\right) \hat{x}_{3}
$$

where $\delta($.$) is the Dirac distribution and \delta^{\prime}($.$) its derivative. The Fourier transform$ of the vorticity must be calculated for a wave vector

$$
q_{i \rightarrow j}=\left(\begin{array}{c}
-\frac{\nu_{0}}{c_{i}}+\frac{\nu}{c_{j}} \cos \theta \\
\frac{\nu}{c_{j}} \sin \theta \\
0
\end{array}\right) ;
$$


its only nonzero component is $\widetilde{\omega}_{s 3}$ and reads

$$
\widetilde{\omega}_{s 3}\left(\mathbf{q}_{i \rightarrow j}, \Delta \nu\right)=\frac{\Gamma L}{(2 \pi)^{3}} \delta\left[\Delta \nu-\left(\frac{\nu_{0}}{c_{i}}-\frac{\nu}{c_{j}}\right) V_{d}\right](-2 i a) \frac{\nu}{c_{j}} \sin \theta
$$

where $L$ is either the length of the dipole of the extension of the sound wave in the $x_{3}$ direction. A factor $a / \lambda_{j}$ appears in $(65)$, which comes from the appearance of a lower wavelength limit in the problem, i.e., the dipole dimension $a$. The following calculations are thus valid for long wavelength $(\lambda \gg a)$, which reinforces the tendency already shown by (62) and (61), to be compared respectively with the explicit calculations (69) and (71).

¿From the expression (65) we can calculate the angular dependence of the scattered waves, using

$$
\begin{aligned}
(\hat{\imath} \times \hat{r}) \cdot \widetilde{\omega}_{s} & =\widetilde{\omega}_{s 3} \sin \theta \\
\hat{r}_{i} \hat{\imath}_{j} \widetilde{\Omega}_{i j} & =-\widetilde{\omega}_{s 3} \cos \theta
\end{aligned}
$$

Since our formulae are valid in three dimensions, it is assumed that any detectors are placed at distances large, compared with $L$, from the vortex dipole.

¿From (42) and (32) we may now calculate the ratio of the total scattered intensity of first and second sound waves when the vortex dipole is illuminated by a second sound wave. It reads

$$
\frac{I_{2 \rightarrow 1}}{I_{2 \rightarrow 2}}=\frac{\rho_{s}^{0} \rho_{n}^{0}}{\rho_{0}^{2}} 4\left(\frac{c_{2}}{c_{1}}\right)^{7} \frac{\int_{0}^{2 \pi} \cos ^{2} \theta \sin ^{4} \theta /\left(1+\alpha^{2}-2 \alpha \cos \theta\right)^{2} \mathrm{~d} \theta}{\int_{0}^{2 \pi}\left(H_{c}(\theta) \sin ^{2} \theta+(B / 2) \sin \theta \cos \theta\right)^{2} \mathrm{~d} \theta}
$$

where $\alpha \equiv c_{2} / c_{1}$ is a small quantity. The integral in the numerator is given in the tables of Gradshteyn and Ryzhik [24] and its value is $\pi / 8$ to leading order in $\alpha$. The numerical value of the integral in the denominator is about $6 \pi$; thus

$$
\frac{I_{2 \rightarrow 1}}{I_{2 \rightarrow 2}}=\frac{\rho_{s}^{0} \rho_{n}^{0}}{\rho_{0}^{2}} \frac{1}{12}\left(\frac{c_{2}}{c_{1}}\right)^{7}
$$


to leading order in $c_{2} / c_{1}$; this is indeed very small, as it was anticipated above on the basis of general considerations. The tendency for most of the energy to be scattered into second sound is increased by the dipolar nature of the target, which implies long wavelength incident waves.

Similarly, from (51) and (47) we may compute the ratio of the total scattered intensity of first and second sound waves when the vortex dipole is illuminated by a second sound wave. The result is

$$
\frac{I_{1 \rightarrow 2}}{I_{1 \rightarrow 1}}=\frac{\rho_{n}^{0}}{\rho_{s}^{0}}\left(\frac{c_{1}}{c_{2}}\right)^{5} \frac{\int_{0}^{2 \pi}\left(\Theta(\theta) \sin ^{2} \theta\right)^{2} \mathrm{~d} \theta}{\int_{0}^{2 \pi} \cos ^{2} \theta(\cos \theta+1)^{2} \mathrm{~d} \theta}
$$

The evaluation of the integral in the numerator is easy to perform with the help of [24]; to leading order in the small quantity $c_{2} / c_{1}$, the ratio reads

$$
\frac{I_{1 \rightarrow 2}}{I_{1 \rightarrow 1}}=\frac{1}{7} \frac{\rho_{n}^{0}}{\rho_{s}^{0}}\left(\frac{c_{1}}{c_{2}}\right)^{3}\left\{3 \frac{T_{0}^{2} S_{0}^{4} \rho_{s}^{0^{2}} \rho_{0}^{2}}{c_{1}^{2} c_{2}^{2} C^{2} \rho_{n}^{0^{2}}}\left[\frac{\rho_{0}}{\rho_{s}^{0}}\left(\frac{\partial \rho_{s}}{\partial \rho}\right)_{T}^{0}-1\right]^{2}+2\right\} .
$$

This quantity may be quite large not too far from the $\lambda$-point (the cumbersome expression in the brackets is of order one). Again, the tendency for most of the energy to be scattered as second sound is increased at long wavelengths in the case of a vortex dipole.

\section{Concluding Remarks}

We have studied the scattering of first and second sound waves by quantum vorticity in superfluid Helium within the context of two fluid hydrodynamics. Within a first

Born approximation explicit expressions have been obtained for the scattering of second sound into both first and second sound (Eqns. (32) and (42)), as well as for the scattering of first sound into both first and second sound (Eqns. (47) and (51)). The relevant formulae coincide with cases already studied (second sound scattered 
into second sound 14, 13] and first sound into first sound 18]) in the appropriate limits. It appears that experimental verification of these effects is not outside the possibilities of currently available hardware 13. In that case, a new tool to study quantum vorticity might become available.

Acknowledgements This work was suppported by ECOS/Conicyt, Fondecyt Grant 1960892 and a Cátedra Presidencial en Ciencias.

\section{References}

[1] C. J. Gorter and J. H. Mellink, Physica 15, 285 (1949).

[2] H. E. Hall and W. F. Vinen, Proc. Roy. Soc A238, 204 (1956).

[3] H. E. Hall and W. F. Vinen, Proc. Roy. Soc A238, 215 (1956).

[4] R. J. Donnelly, Quantized Vortices in Helium II, (Cambridge University Press, Cambridge, 1991).

[5] E. B. Sonin, Rev. Mod. Phys. 59, 87 (1987).

[6] Q. Niu, P. Ao and D. J. Thouless, Phys. Rev. Lett. 72, 1706 (1994); Phys. Rev. Lett 75, 1706 (1995).

[7] J.-M. Duan and A. J. Legget, Phys. Rev. Lett. 68, 1216 (1992); J.-M. Duan, Phys. Rev. B 48, 333 (1993); Phys. Rev. B 49, 12381 (1994); Phys. Rev. Lett. 75,974 (1995).

[8] D. J. Thouless, P. Ao and Q. Niu, Phys. Rev. Lett 76, 1706 (1996).

[9] E. Demircan, P. Ao and Q. Niu, Phys. Rev. B, 52, 476 (1995). 
[10] C. Nore, M. E. Brachet, E. Cerda and E. Tirapegui, Phys. Rev. Lett. 72, 2593 (1994).

[11] E. Demircan, P. Ao and Q. Niu, Phys. Rev. B, 54, 10027 (1996).

[12] I. M. Khalatnikov, Introduction to the Theory of Superfluidity, (Benjamin, New York, 1965).

[13] F. Lund and V. Steinberg, Phys. Rev. Lett., 75, 1102 (1995).

[14] L. P. Pitaevskii. Sov. Phys. JETP 35, 888 (1959).

[15] A. Fetter, Phys. Rev. A 136, 1488 (1964).

[16] S. K. Nemirovskii, Sov. Phys. Usp. 33, 429 (1990).

[17] L. C. Krysac, Phys. Rev. Lett. 73, 2480 (1994).

[18] F. Lund and C. Rojas, Physica D, 37, 508 (1989).

[19] C. Baudet, S. Ciliberto and J.-F. Pinton, Phys. Rev. Lett. 67, 193 (1991).

[20] L. D. Landau and E. M. Lifshitz, Fluid Mechanics (Pergamon Press, London, 1987).

[21] W. Möhring, J. Fluid Mech. 85, 685 (1978).

[22] R. Berthet and F. Lund, Phys. Fluids 7(10), 2522 (1995).

[23] H. Lamb, Hydrodynamics, (Cambridge University Press, Cambridge, 1975).

[24] I.S. Gradsteyn and I.M. Ryzhik, Tables of integrals, series and products, (Academic Press, New York, 1994). 